\title{
Avaliação do bem-estar animal nas operações de insensibilização e sangria de bovídeos em abatedouros no Amazonas, Brasil
}

\section{Evaluation of animal welfare in insensibilization and bleeding of cattle in slaughterhouses in Amazonas, Brazil}

DOI: $10.46919 / \operatorname{arch} 2 \mathrm{n} 1-005$

Recebimento dos originais: 30/10/2020

Aceitação para publicação: 23/12/2020

\section{Ana Paula Assis Sá \\ Graduada em Medicina Veterinária}

Instituição: Instituto Federal de Educação, Ciência e Tecnologia do Estado do Amazonas (IFAM),

Campus Manaus Zona Leste (CMZL)

Endereço: Avenida Cosme Ferreira, nº. 8.045, Bairro: São José Operário, Manaus/AM

E-mail: anapsa23@gmail.com

\section{Jomel Francisco dos Santos}

Doutor em Ciência Veterinária pela Universidade Federal Rural de Pernambuco

Instituição: Instituto Federal de Educação, Ciência e Tecnologia do Estado do Amazonas (IFAM),

Campus Manaus Zona Leste (CMZL)

Endereço: Avenida Cosme Ferreira, nº. 8.045, Bairro: São José Operário, Manaus/AM

E-mail: jomel.santos@ifam.edu.br

\section{Eduardo Lima de Sousa}

Mestre em Educação Agrícola pela Universidade Federal Rural do Rio de Janeiro Instituição: Instituto Federal de Educação, Ciência e Tecnologia do Estado do Amazonas (IFAM),

Campus Manaus Zona Leste (CMZL)

Endereço: Avenida Cosme Ferreira, nº. 8.045, Bairro: São José Operário, Manaus/AM

E-mail: eduardo.sousa@ifam.edu.br

\section{Edson Francisco do Espírito Santo}

Doutor em Ciência Animal pela Universidade Estadual Paulista "Júlio de Mesquita Filho" Instituição: Instituto Federal de Educação, Ciência e Tecnologia do Estado do Amazonas (IFAM),

Campus Manaus Zona Leste (CMZL)

Endereço: Avenida Cosme Ferreira, nº. 8.045, Bairro: São José Operário, Manaus/AM

E-mail: edson.santo@ifam.edu.br

\section{RESUMO}

O procedimento de abate de bovídeos vem se modificando e se modernizando com o passar dos anos, com o intuito de satisfazer um mercado consumidor cada vez mais preocupado com o bem-estar animal e com o desenvolvimento sustentável. O objetivo deste trabalho foi avaliar o bem-estar animal nas etapas de insensibilização e sangria em três abatedouros-frigoríficos na região metropolitana de Manaus-AM. Acompanhou-se o abate de 300 bovídeos, com diferentes faixas etárias, raças e sexos, onde considerou-se a eficiência do atordoamento, em função do número de disparos da pistola pneumática por animal, e o monitoramento dos intervalos dos seguintes tempos: entre a insensibilização e a sangria, entre o início da sangria e o corte de patas, e de permanência dos animais na calha de sangria. Ao considerar cada lote com 
100 animais, o percentual de indivíduos insensibilizados com apenas um disparo ficou entre $27 \%$ e $67 \%$, conferindo a classificação de problema sério, nos três estabelecimentos avaliados. O tempo médio entre a insensibilização e a sangria oscilou entre 1 min e 10 s e 2 min 52 s. Nas análises feitas na calha, apenas o abatedouro 2 respeitou o tempo mínimo de sangria, de $3 \mathrm{~min}$, sendo que nos outros dois locais visitados, observou-se a realização de mutilações nos animais ainda vivos. O tempo médio de permanência dos animais na calha variou de 2 min e $34 \mathrm{~s}$ a 4 min e $54 \mathrm{~s}$. Os resultados não satisfatórios encontrados nos abatedouros revelam que os mesmos não possuem um plano de abate humanitário, causando sofrimento desnecessário aos animais durante esse procedimento. Dessa forma, é extremamente importante salientar a necessidade de capacitação dos funcionários a respeito do assunto e de alterações na infraestrutura para obtenção de melhores índices de bem-estar animal e consequentemente de uma carne com melhor qualidade.

Palavras-chave: abate humanitário, atordoamento, boas práticas de produção, matadouro, qualidade da carne.

\begin{abstract}
The procedure for slaughtering cattle has been changing and modernizing over the years in order to satisfy a consumer market increasingly concerned with animal welfare and sustainable development. The objective of this work was to evaluate animal welfare in the stages of insensitization and bleeding in three slaughterhouses in the metropolitan region of Manaus-AM. The slaughter of 300 cattle, with different age groups, races and sexes, was followed, where the efficiency of the stunning was considered, according to the number of shots of the pneumatic gun per animal, and the monitoring of the intervals of the following times: between insensitization and bleeding, between the beginning of bleeding and the cutting of legs, and the permanence of the animals in the bleeding gutter. When considering each flock with 100 animals, the percentage of insensitized individuals with only one shot was between $27 \%$ and $67 \%$, conferring the classification of a serious problem in the three evaluated establishments. The average time between insensitization and bleeding oscillated between $1 \mathrm{~min}$ and $10 \mathrm{~s}$ and $2 \mathrm{~min} 52 \mathrm{~s}$. In the analyses made in the trough, only the slaughterhouse 2 respected the minimum time of bleeding, of $3 \mathrm{~min}$, being that in the other two places visited, it was observed the accomplishment of mutilations in the still alive animals. The average time the animals stayed in the trough varied from $2 \mathrm{~min}$ and $34 \mathrm{~s}$ to $4 \mathrm{~min}$ and $54 \mathrm{~s}$. The unsatisfactory results found in the slaughterhouses reveal that they do not have a humanitarian slaughter plan, causing unnecessary suffering to the animals during this procedure. Therefore, it is extremely important to emphasize the need for training of employees on the subject and changes in infrastructure to obtain better rates of animal welfare and consequently better quality meat.
\end{abstract}

Keywords: humanitarian slaughter, stunning, good production practices, slaughterhouse, meat quality.

\title{
1 INTRODUÇÃO
}

Por muito tempo, o processo de abate dos animais foi considerado uma operação técnica de baixo nível científico, não constituindo uma temática comumente estudada. No entanto, tem-se atribuído grande importância ao assunto em decorrência das perdas econômicas no setor e da preocupação com o sofrimento dos animais destinados ao abate, levando-se em consideração toda a cadeia produtiva, desde as condições de criação nas fazendas, o transporte, a chegada aos estabelecimentos industriais até o momento da morte efetiva, observando-se as condições físicas e sensitivas desses indivíduos (GUERRERO et al., 2013; DUARTE e AFONSO, 2020). 
Atualmente, as responsabilidades em torno do bem-estar animal e do abate humanitário se traduzem em normatizações técnicas, institucionalizadas, nacional e internacionalmente, do ponto de vista científico e das leis, obrigando os profissionais e a indústria a se adaptarem a um novo universo de cuidados e buscarem novas estratégias com o intuito de reordenar em termos morais e técnicos a sua forma de atuação (PERROTA, 2016). Além disso o avanço dessa ciência aguçou o senso crítico da necessidade de prevenção do sofrimento animal, adicionado ao olhar atento do consumidor, às boas práticas de produção e a preservação ambiental, sendo que o bem-estar animal agregou valor ao produto favorecendo a sua produtividade (BRAGA et al., 2018).

A primeira e mais crítica etapa do abate de bovídeos consiste na insensibilização, processo que deixa o animal desfalecido para a realização de uma sangria mais eficiente, sem causar dor ao animal (SOBRAL et al., 2015). Essas etapas do abate requerem muita atenção, sendo necessárias instalações adequadas, equipamentos devidamente calibrados e mão-de-obra qualificada para sua realização (LANDIM, 2011).

Um manejo inadequado durante as etapas do pré-abate e abate representa, além de estresse aos animais, prejuízos econômicos com contusões nas carcaças e alterações químicas, fisiológicas e biológicas da carne, aumentando o seu $\mathrm{pH}$, deixando-a mais escura, tornando-a desagradável ao consumidor e consequentemente resultando em perda de produção ou venda de produtos de baixa qualidade (LEITE et al., 2015; ALVES et al., 2016). Pensando nisso, este estudo objetivou avaliar a eficiência das operações de insensibilização e sangria sob a perspectiva do bem-estar animal, em três abatedouros no estado do Amazonas, Brasil, com Serviço de Inspeção Estadual.

\section{MATERIAL E MÉTODOS}

A coleta de dados foi realizada em três abatedouros-frigoríficos no ano de 2019, na região metropolitana de Manaus. O abate médio diário dos estabelecimentos era de 300 animais, sendo machos e fêmeas de diferentes categorias e raças, que estavam sob supervisão do Serviço de Inspeção Estadual. As análises nos locais foram feitas observando-se as etapas de insensibilização e sangria, onde foram avaliados: a eficácia da insensibilização; o tempo entre a insensibilização e a sangria; o tempo entre o início da sangria e corte de patas, e o tempo de permanência dos animais na calha de sangria, utilizando-se lotes de 100 animais.

A eficácia da insensibilização foi avaliada pela porcentagem de animais atordoados com apenas um disparo de pistola pneumática, seguindo a metodologia de Grandin (2003), considerando-se: "Excelente" o lote onde $99 \%$ a $100 \%$ dos animais foram insensibilizados com apenas um disparo; "Aceitável” - o lote onde 95\% a 98\% dos animais foram insensibilizados com apenas um disparo; "Não aceitável” - o lote onde 90\% a 94\% dos animais foram insensibilizados com apenas um disparo; "Problema sério" - o lote onde menos de $90 \%$ dos animais foram insensibilizados com apenas um disparo. 
O tempo entre a insensibilização e a sangria foi avaliado levando-se em consideração o que é preconizado no Regulamento Técnico de métodos de insensibilização para o abate humanitário de animais de açougue (BRASIL, 2000), o qual estabelece que este intervalo deve ser de no máximo um min. Em relação ao tempo de sangria, este foi avaliado conforme o prescrito no documento de Padronização de Técnicas, Instalações e Equipamentos de bovinos do Ministério da Agricultura, Pecuária e Abastecimento, cujo tempo mínimo estimado dever ser de três min (BRASIL, 2007). Cronometrou-se, então, o tempo desde o momento do corte dos grandes vasos do pescoço até o corte das patas e o tempo de permanência dos animais na calha de sangria.

Os dados coletados foram organizados em planilhas no Microsoft EXCEL® Office 2016, realizando então a confecção do banco de dados, os quais posteriormente foram organizados em tabelas e gráficos segundo as variáveis utilizadas. A abordagem da pesquisa realizada foi quantitativa, sendo que para análise dos dados utilizou-se a estatística descritiva.

\section{RESULTADOS E DISCUSSÃO}

\subsection{AVALIAÇÃO DA EFICIÊNCIA DA INSENSIBILIZAÇÃO}

Segundo Brasil (2000), a insensibilização é um processo aplicado no abatedouro-frigorífico para proporcionar um estado de rápida inconsciência e insensibilidade ao animal, mantendo as suas funções vitais até a operação de sangria. Nos estabelecimentos analisados, o método de insensibilização aplicado é o mecânico, percussivo penetrativo, com o uso de uma pistola com dardo cativo. No momento desta operação, a pistola deve ser posicionada de modo a garantir que o dardo penetre o córtex cerebral, através da região frontal (BRASIL, 2000), na intersecção de duas linhas imaginárias, que vão da base do chifre até o olho do lado oposto na cabeça do animal (NEVES, 2008). Na Figura 1, ilustra-se a realização desse processo no abatedouro 2.

Figura 1 - Insensibilização, abatedouro 2.

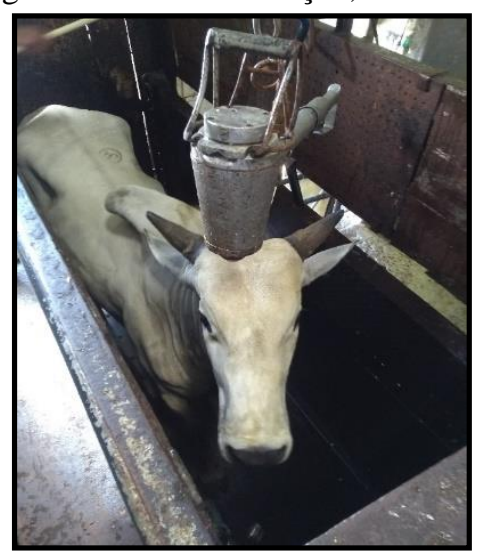

Fonte: Autoria Própria 
Para a avaliação da eficácia da insensibilização, contabilizou-se o número de disparos da pistola necessários para atordoar um animal. Os resultados obtidos nos três abatedouros-frigoríficos avaliados estão dispostos na Tabela 1 e ilustrados em gráficos nas Figuras 2, 3 e 4.

Tabela 1 - Avaliação da eficácia da insensibilização em função do número de disparos da pistola por animal.

\begin{tabular}{c|ccc}
\hline $\mathbf{N}^{\circ}$ DE DISPAROS & AF 1 & AF 2 & AF 3 \\
\hline $\mathbf{1}$ DISPARO & 27 & 67 & 31 \\
$\mathbf{2}$ DISPAROS & 54 & 23 & 37 \\
3 OU + DISPAROS & 19 & 10 & $\mathbf{1 0 0}$ \\
\hline
\end{tabular}

Legenda: AF - Abatedouro-Frigorífico

Figura 2 - Percentual de animais insensibilizados em relação ao número de disparos realizados por pistola pneumática penetrativa, abatedouro 1 .

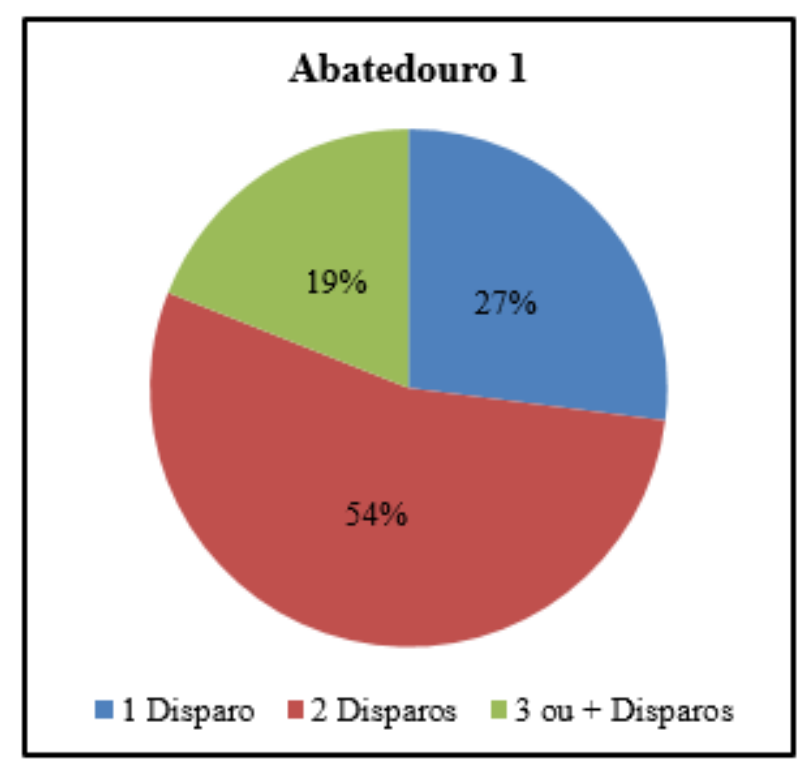

Figura 3 - Percentual de animais insensibilizados em relação ao número de disparos realizados por pistola pneumática penetrativa, abatedouro 2 .

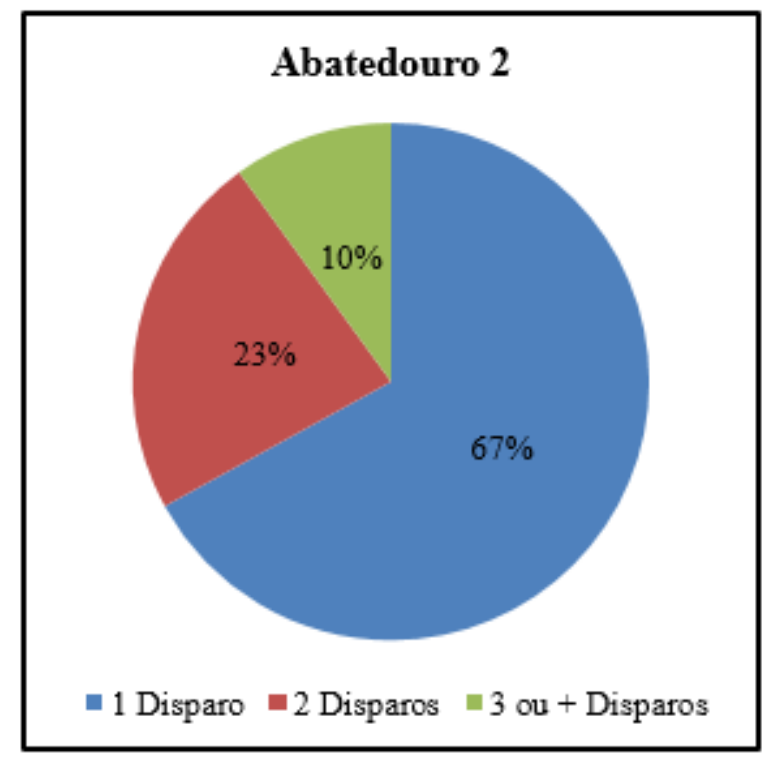


Figura 4 - Percentual de animais insensibilizados em relação ao número de disparos realizados por pistola pneumática penetrativa, abatedouro 3 .

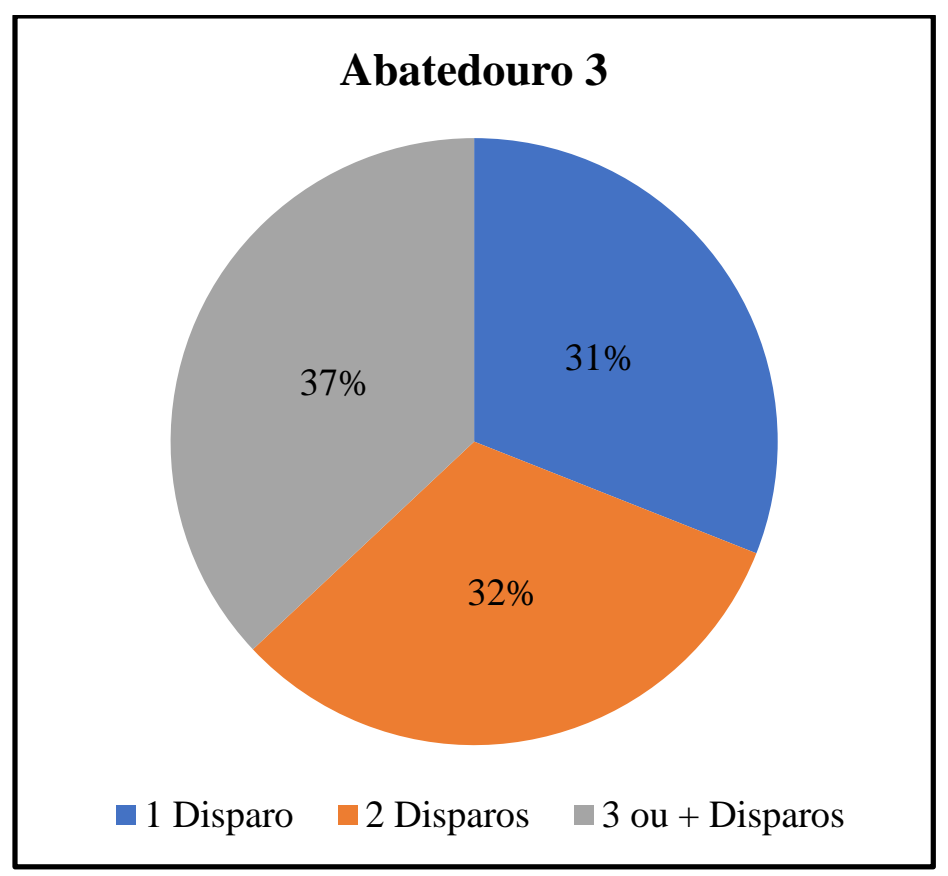

Segundo Grandin (2003), se 99\% a 100\% dos animais de um lote são insensibilizados com apenas um disparo, esta operação pode ser considerada como "excelente", se 95\% a 98\% pode ser considerada como "aceitável", se $90 \%$ a 94\% como "não aceitável", e se abaixo de 90\% como "problema sério". Observando-se as análises feitas, nota-se que o percentual de animais insensibilizados com apenas um disparo ficou abaixo dos 90\%, conferindo a classificação de "problema sério", de acordo com tal metodologia.

As causas mais comuns de um baixo índice na eficácia da insensibilização provêm principalmente de manutenção deficiente do equipamento e falta de treinamento, experiência ou cansaço dos funcionários que realizam essa operação (GRANDIN, 2003), problema esse identificado nos locais visitados já que um único colaborador exercia essa função, sem rotação de atividade. Além disso, nos abatedouros 1 e 3, os bovídeos eram colocados em duplas dentro do boxe de insensibilização (Figuras 5 e 6), ficando bastante inquietos durante essa operação, quando frequentemente se agitavam e se movimentavam, fazendo com que o operador efetuasse dois, três e em algumas situações, mais de três disparos na cabeça do animal. Resultados semelhantes foram encontrados por Mártire (2016) e Carlesci et al. (2014) em seus estudos, onde apenas $69 \%$ e $70 \%$ dos animais avaliados, respectivamente, foram insensibilizados com um único disparo da pistola.

O box de atordoamento dos locais estudados era do tipo convencional, sem fixação da cabaça do animal, no entanto, Bertoloni e Andreolla (2010) constataram que o box automatizado com contenção de 
cabeça permitiu uma melhoria na eficácia da insensibilização de bovinos abatidos no estado do Mato Grosso

- Brasil, por auxiliar o operador a posicionar corretamente a cabeça do animal

na hora do disparo. Contudo, destacam também que a capacitação dos operadores para utilização dos equipamentos é indispensável para obtenção de índices melhores.
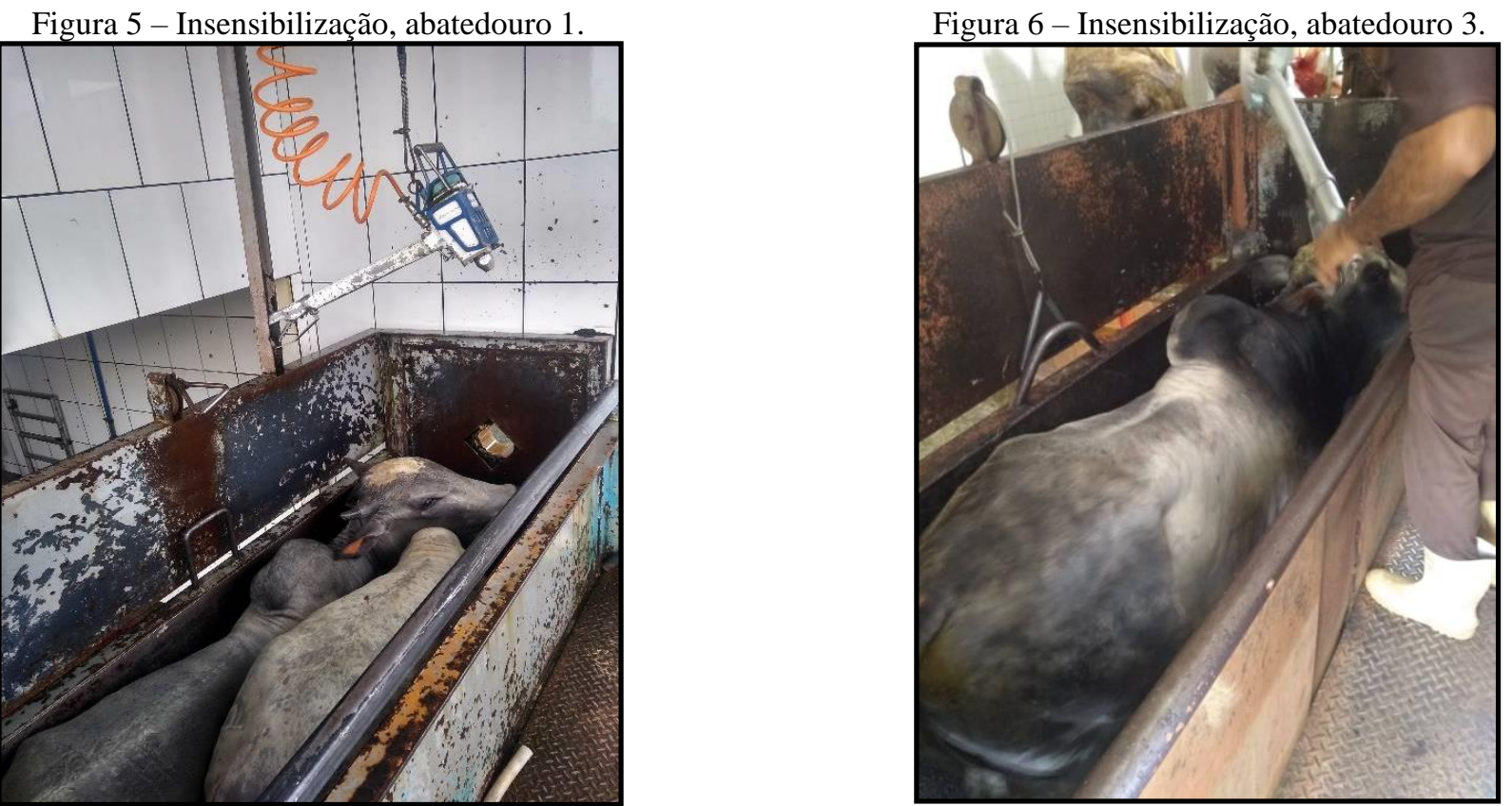

\subsection{AVALIAÇÃO DO TEMPO ENTRE A INSENSIBILIZAÇÃO E A SANGRIA}

Após a insensibilização, a operação de sangria deverá ser iniciada o mais rápido possível, em no máximo $1 \mathrm{~min}$, antes que o animal recupere a sensibilidade (BRASIL, 2000). Os resultados para a avaliação do tempo entre a insensibilização e sangria nos locais avaliados, encontram-se descritos na Tabela 2

\begin{tabular}{|c|c|c|c|c|}
\hline $\begin{array}{l}\text { Abatedouro- } \\
\text { frigorífico }\end{array}$ & Tempo mínimo & Tempo máximo & Média & Desvio Padrão \\
\hline 1 & $58 \mathrm{~s}$ & $\begin{array}{c}412 \mathrm{~s} \\
(6 \min 52 \mathrm{~s})\end{array}$ & $\begin{array}{c}172,51 \mathrm{~s} \\
(2 \min 52 \mathrm{~s})\end{array}$ & 49,2 \\
\hline 2 & $35 \mathrm{~s}$ & $\begin{array}{c}235 \mathrm{~s} \\
(3 \min 55 \mathrm{~s})\end{array}$ & $\begin{array}{c}70,74 \mathrm{~s} \\
(1 \mathrm{~min} 10 \mathrm{~s})\end{array}$ & 24,7 \\
\hline 3 & $10 \mathrm{~s}$ & $\begin{array}{c}483 \mathrm{~s} \\
(8 \min 3 s)\end{array}$ & $\begin{array}{c}101,97 \mathrm{~s} \\
(1 \min 41 \mathrm{~s})\end{array}$ & 78,4 \\
\hline
\end{tabular}

É possível notar que a amplitude dos dados é muito grande nos três abatedouros, tendo essa variação demonstrada pelo desvio padrão. Isso é fruto de uma variável com o máximo muito distante do mínimo, provando que nos estabelecimentos visitados não havia um padrão de tempo a ser obedecido. Estes resultados se assemelham ao descrito por Gallo et al. (2003), onde os valores oscilaram entre 1 e 4 min, sendo estes índices melhorados após a capacitação dos funcionários. Por outro lado, Leite et al. (2015) 
obtiveram resultados satisfatórios, com todos os valores médios observados permanecendo dentro do tempo máximo permitido de um min.

Analisando-se os dados contidos na Tabela 3 e na Figura 7, é possível observar que no abatedouro 1, apenas um animal dos 100 avaliados, respeitou o tempo de um min, estipulado no Regulamento Técnico de métodos de insensibilização para abate humanitário de animais de açougue (2000), havendo animais que chegaram a ultrapassar 6 min.

Tabela 3 - Distribuição de frequência de tempo entre insensibilização e sangria, abatedouro 1.

\begin{tabular}{|c|c|c|}
\hline $\begin{array}{l}\text { Classes de Tempo Entre } \\
\text { Insensibilização e Sangria }\end{array}$ & Frequência Absoluta & Frequência Relativa \\
\hline 0 F60 & 1 & $1 \%$ \\
\hline $60 F 120$ & 6 & $6 \%$ \\
\hline $120 \mathrm{~F} 180$ & 54 & $54 \%$ \\
\hline 180 F 240 & 33 & $33 \%$ \\
\hline $240 F 300$ & 4 & $4 \%$ \\
\hline $300 \div 360$ & 1 & $1 \%$ \\
\hline $360+420$ & 1 & $1 \%$ \\
\hline TOTAL & 100 & $100 \%$ \\
\hline
\end{tabular}

Figura 7 - Distribuição de frequência de tempo entre a insensibilização e a sangria, abatedouro 1.

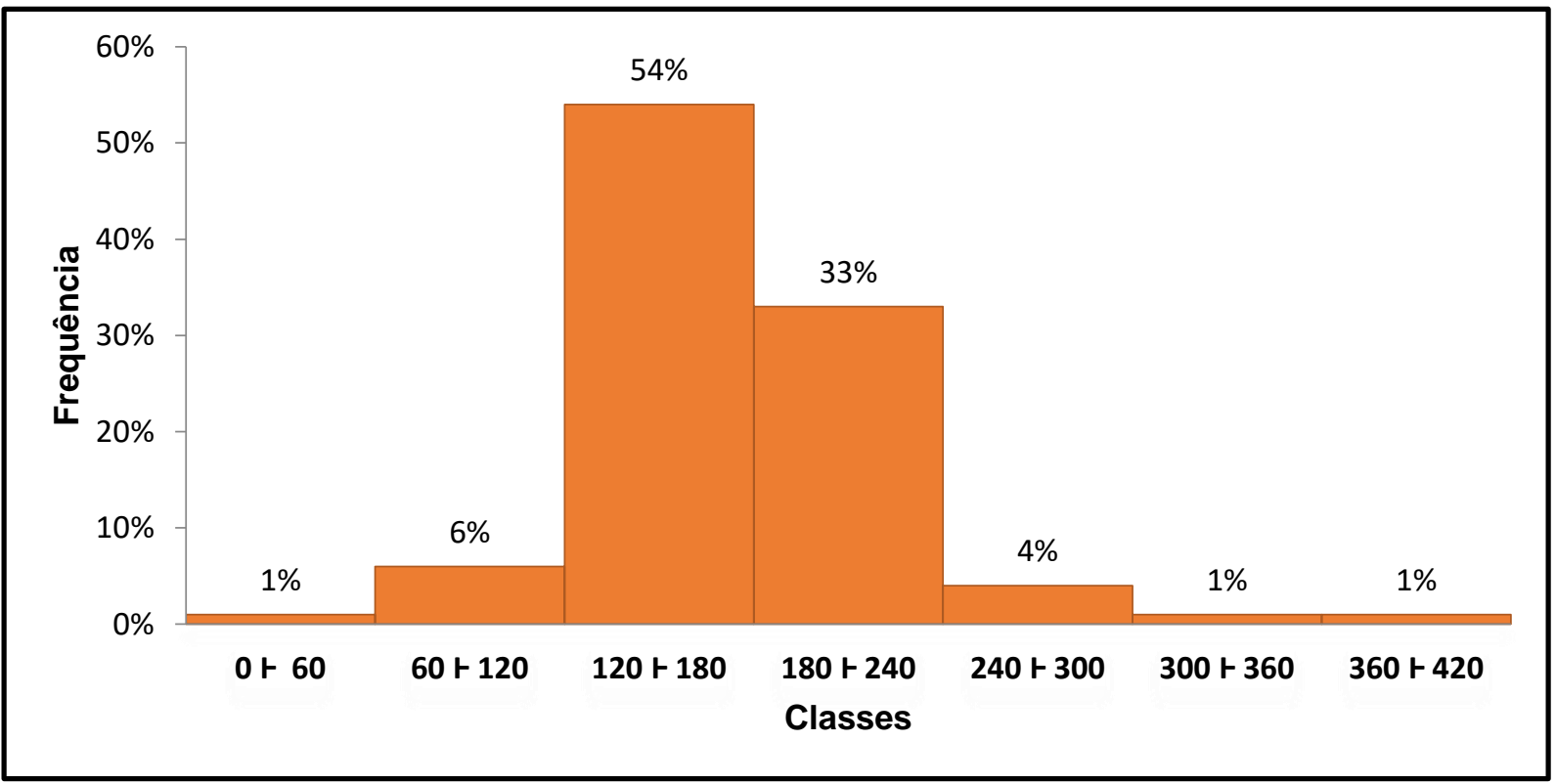

Neste abatedouro os colaboradores demonstraram desconhecer que havia um tempo máximo entre as operações de insensibilização e sangria, pois não apresentaram preocupação em realizar os procedimentos da forma mais rápida possível. Além disso, muitas vezes utilizavam este intervalo para amolar suas facas ou se higienizar. 
No abatedouro 2, segundo os dados contidos na Tabela 4 e Figura 8, nota-se que o número de animais que se enquadram no intervalo é bem maior que o observado no abatedouro 1, porém ainda corresponde a menos da metade dos animais avaliados.

Tabela 4 - Distribuição de frequência de tempo entre insensibilização e sangria, abatedouro 2.

\begin{tabular}{c|cc}
\hline $\begin{array}{c}\text { Classes de Tempo Entre } \\
\text { Insensibilização e Sangria }\end{array}$ & Frequência Absoluta & Frequência Relativa \\
\hline O F $\mathbf{6 0}$ & 33 & $33 \%$ \\
$\mathbf{6 0}$ H $\mathbf{1 2 0}$ & 63 & $63 \%$ \\
$\mathbf{1 2 0}$ H $\mathbf{1 8 0}$ & 3 & $3 \%$ \\
$\mathbf{1 8 0}$ 240 & 1 & $1 \%$ \\
\hline TOTAL & $\mathbf{1 0 0}$ & $\mathbf{1 0 0} \%$ \\
\hline
\end{tabular}

Figura 8 - Distribuição de frequência de tempo entre a insensibilização e a sangria, abatedouro 2.

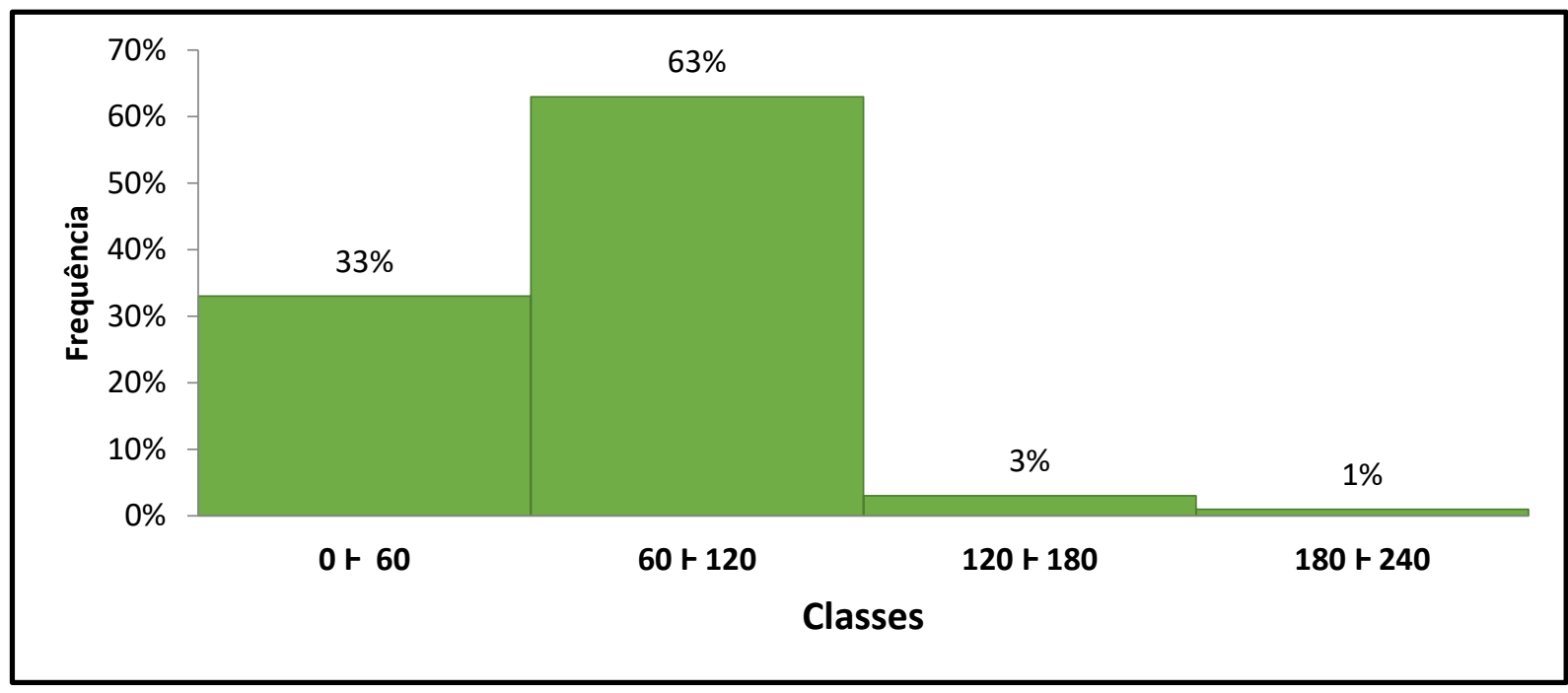

Neste estabelecimento notou-se atraso no ato de alçar os animais, e que o equipamento utilizado para tal ação, comumente apresentava problemas no dia da análise, o que resultou em uma demora neste intervalo de tempo.

No abatedouro 3, através dos dados contidos na Tabela 5 e na Figura 9, observa-se que o quantitativo de animais que se enquadraram no tempo correto entre a insensibilização e a sangria também corresponde a menos da metade dos animais avaliados. No local, também se registrou o maior intervalo de tempo, sendo de 9 min. 
Figura 9 - Distribuição de frequência de tempo entre a insensibilização e a sangria, abatedouro 3.

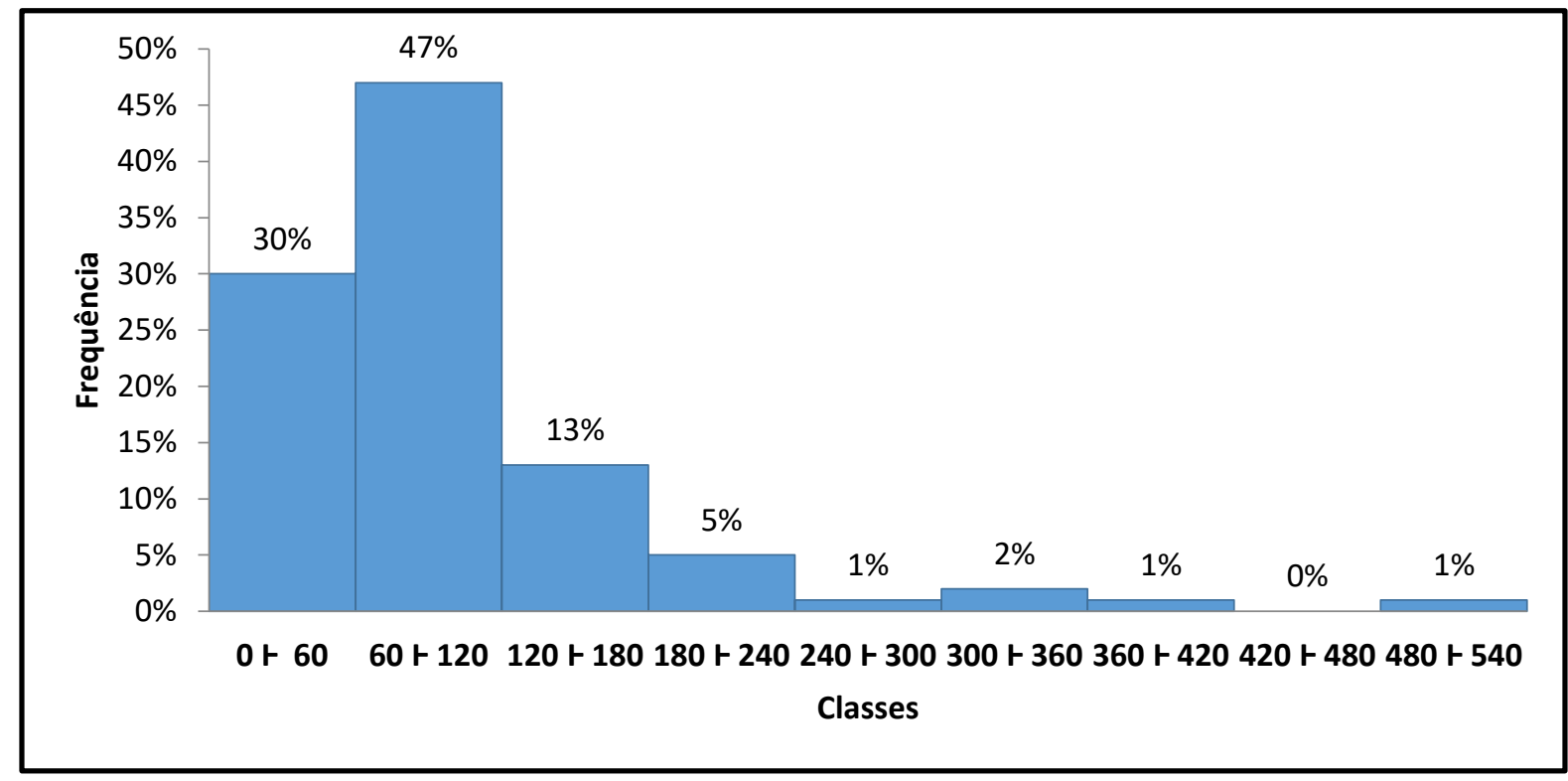

Tabela 5 - Distribuição de frequência de tempo entre insensibilização e sangria, abatedouro 3.

\begin{tabular}{|c|c|c|}
\hline $\begin{array}{l}\text { Classes de Tempo entre } \\
\text { Insensibilização e Sangria }\end{array}$ & Frequência Absoluta & Frequência Relativa \\
\hline $0 \mathrm{~F} 60$ & 30 & $30 \%$ \\
\hline 60 F 120 & 47 & $47 \%$ \\
\hline $120+180$ & 13 & $13 \%$ \\
\hline 180 F 240 & 5 & $5 \%$ \\
\hline $240+300$ & 1 & $1 \%$ \\
\hline $300+360$ & 2 & $2 \%$ \\
\hline $360+420$ & 1 & $1 \%$ \\
\hline $420+480$ & 0 & $0 \%$ \\
\hline 480 F 540 & 1 & $1 \%$ \\
\hline TOTAL & 100 & $100 \%$ \\
\hline
\end{tabular}

Nesse estabelecimento, a demora neste intervalo se deu por fatores semelhantes aos observados no abatedouro 1, os colaboradores constantemente se distraíam com outras atividades e demoravam para iniciar a sangria.

\subsection{AVALIAÇÃO DO TEMPO ENTRE O INÍCIO DA SANGRIA E O CORTE DE PATAS}

A sangria é a operação que consiste na secção dos grandes vasos do pescoço, à altura da entrada do peito, depois de aberta sagitalmente a barbela pela "línea Alba". O tempo mínimo exigido para uma boa sangria é de três min, antes do qual não serão permitidas operações que envolvam mutilações no animal (BRASIL, 2007). Os resultados obtidos para a avaliação do tempo entre o início da sangria e o corte de patas encontram-se descritos nas Tabelas 6 e 7. 
Tabela 6 - Avaliação dos tempos máximos e mínimos aferidos entre o início da sangria e o corte de patas.

\begin{tabular}{|c|c|c|}
\hline Abatedouro-Frigorífico & Tempo Mínimo & Tempo Máximo \\
\hline 1 & $1 \mathrm{~s}$ & $\begin{array}{c}125 \mathrm{~s} \\
(2 \min 5 \mathrm{~s})\end{array}$ \\
\hline 2 & $\begin{array}{c}150 \mathrm{~s} \\
(2 \min 30 \mathrm{~s})\end{array}$ & $\begin{array}{c}295 \mathrm{~s} \\
(4 \min 55 \mathrm{~s})\end{array}$ \\
\hline 3 & 0 & $\begin{array}{c}174 s \\
(2 \min 54 s)\end{array}$ \\
\hline
\end{tabular}

Tabela 7 - Avaliação dos tempos conformes e não-conformes aferidos entre o início da sangria e o corte de patas.

\begin{tabular}{c|cc}
\hline Abatedouro-Frigorífico & Tempos Conformes & Tempos Não-Conformes \\
\hline $\mathbf{1}$ & 0 & 100 \\
$\mathbf{2}$ & 83 & 17 \\
$\mathbf{3}$ & 0 & 100 \\
\hline
\end{tabular}

Nos abatedouros 1 e 3 o tempo de sangria não foi respeitado, sendo que logo após a secção dos grandes vasos do pescoço foram realizadas operações na calha da sangria, que envolviam mutilações nos indivíduos, ainda vivos. No abatedouro-frigorífico 3, flagrou-se inclusive, mutilações em dois bovinos (corte das patas) antes mesmo da operação de sangria ser iniciada. $\mathrm{O}$ abatedouro 2 obteve o melhor resultado para a avaliação desta variável, respeitando o tempo mínimo de 3 min em 83\% dos animais abatidos.

\subsection{AVALIAÇÃO DO TEMPO DE PERMANÊNCIA DOS ANIMAIS NA CALHA DE SANGRIA.}

Como em dois, dos três locais visitados, observou-se que os colaboradores efetuavam mutilações nos animais logo após o início da sangria, aferiu-se também o tempo de permanência dos animais na calha, afim de melhor se avaliar a operação de sangria. Os resultados para a avaliação desta variável, encontramse descritos na Tabela 8.

Tabela 8 - Variação do tempo de permanência dos animais na calha de sangria nos três abatedouros-frigoríficos avaliados.

\begin{tabular}{c|cccc}
\hline $\begin{array}{c}\text { Abatedouro- } \\
\text { Frigorífico }\end{array}$ & Tempo Mínimo & Tempo Máximo & Média & Desvio Padrão \\
\hline $\mathbf{1}$ & $96 \mathrm{~s}$ & $234 \mathrm{~s}$ & $154,09 \mathrm{~s}$ & 28,8 \\
\multirow{2}{2}{} & $(1 \min 36 \mathrm{~s})$ & $(3 \min 54 \mathrm{~s})$ & $(2 \min 34 \mathrm{~s})$ & 37,7 \\
\multirow{3}{*}{} & $227 \mathrm{~s}$ & $399 \mathrm{~s}$ & $294,33 \mathrm{~s}$ & 37,6 \\
\hline
\end{tabular}

Por meio dos dados obtidos para essa análise, é possível observar que muitos indivíduos foram mal sangrados, saindo da calha antes mesmo de se completarem os três min. Resultados semelhantes foram encontrados por Pinto (2018), onde ao avaliar-se as médias ao longo de um período de 12 meses, notou-se que nenhuma se enquadrava dentro do tempo mínimo preconizado pela legislação. 
Observando-se os dados do abatedouro 1, na Tabela 9 e na Figura 10, nota-se que a maioria dos animais permaneceu na calha de sangria pelo intervalo de dois a três min. Cerca de $89 \%$ dos animais saíram da calha durante a realização do processo de sangria, ou imediatamente após a realização da mesma.

Tabela 9 - Intervalos de permanência na calha da sangria, abatedouro 1.

\begin{tabular}{c|cc}
\hline $\begin{array}{c}\text { Classes do Tempo de } \\
\text { Permanência na Calha }\end{array}$ & Frequência Absoluta & Frequência Relativa \\
\hline 0 F $\mathbf{6 0}$ & 0 & $0 \%$ \\
$\mathbf{6 0}$ F $\mathbf{1 2 0}$ & 17 & $17 \%$ \\
$\mathbf{1 2 0}$ F $\mathbf{1 8 0}$ & 72 & $72 \%$ \\
$\mathbf{1 8 0}$ F $\mathbf{2 4 0}$ & 11 & $11 \%$ \\
\hline TOTAL & $\mathbf{1 0 0}$ & $\mathbf{1 0 0} \%$ \\
\hline
\end{tabular}

Figura 10 - Distribuição de frequência do tempo de permanência dos animais na calha da sangria, abatedouro 1.

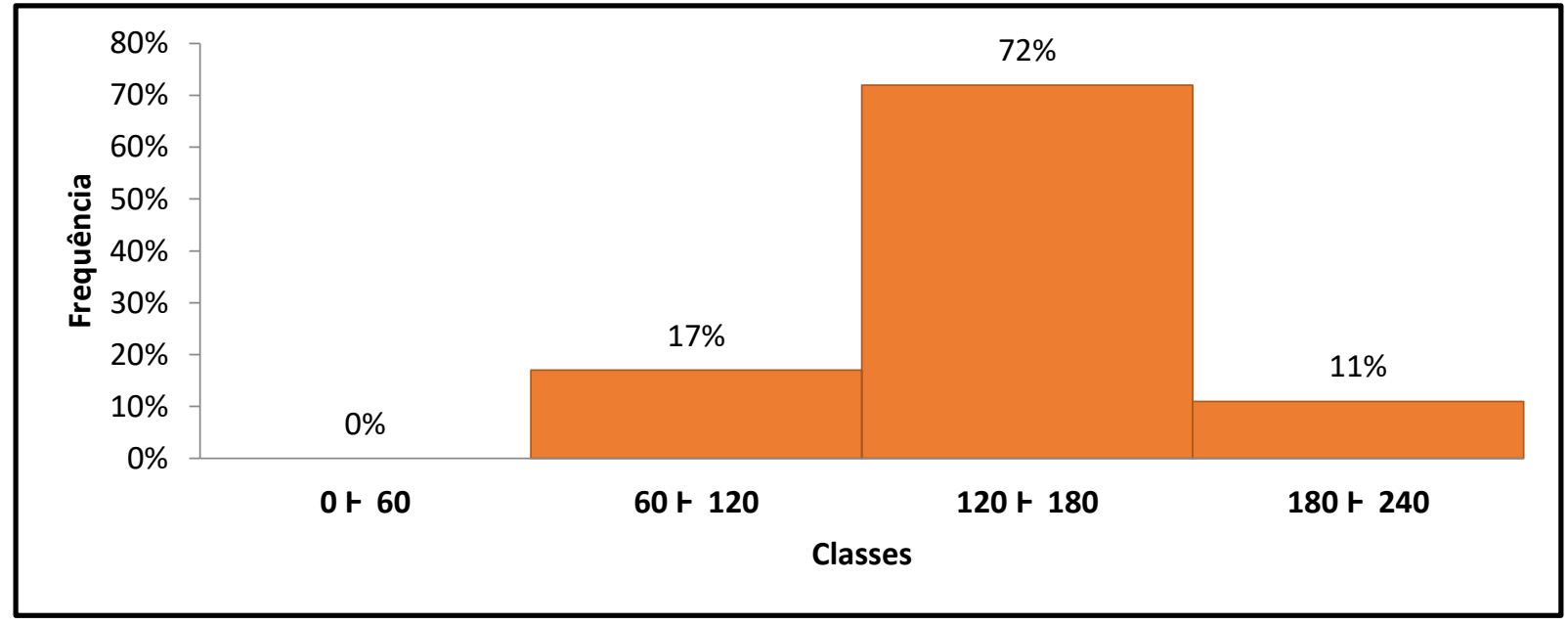

No abatedouro 2, observando-se os dados contidos na Tabela 10 e na Figura 11, nota-se que a maioria dos animais avaliados permaneceu pelo intervalo de 4 a 6 min na calha, já que os operadores de sangria costumavam esperar o tempo mínimo de $3 \mathrm{~min}$, como citado anteriormente.

Tabela 10 - Intervalos de permanência na calha da sangria, abatedouro 2

\begin{tabular}{c|cc}
\hline $\begin{array}{c}\text { Classes do Tempo de Permanência } \\
\text { na Calha }\end{array}$ & Frequência Absoluta & Frequência Relativa \\
\hline $\mathbf{0}$ F $\mathbf{6 0}$ & 0 & $0 \%$ \\
$\mathbf{6 0}$ F $\mathbf{1 2 0}$ & 0 & $0 \%$ \\
$\mathbf{1 2 0}$ F $\mathbf{1 8 0}$ & 0 & $0 \%$ \\
$\mathbf{1 8 0}$ F $\mathbf{2 4 0}$ & 6 & $6 \%$ \\
$\mathbf{2 4 0}$ H 300 & 54 & $54 \%$ \\
$\mathbf{3 0 0}$ F $\mathbf{3 6 0}$ & 33 & $33 \%$ \\
$\mathbf{3 6 0}$ H $\mathbf{4 2 0}$ & 7 & $7 \%$ \\
\hline TOTAL & $\mathbf{1 0 0}$ & $\mathbf{1 0 0} \%$ \\
\hline
\end{tabular}


Figura 11 - Distribuição de frequência do tempo de permanência dos animais na calha da sangria, abatedouro 2.

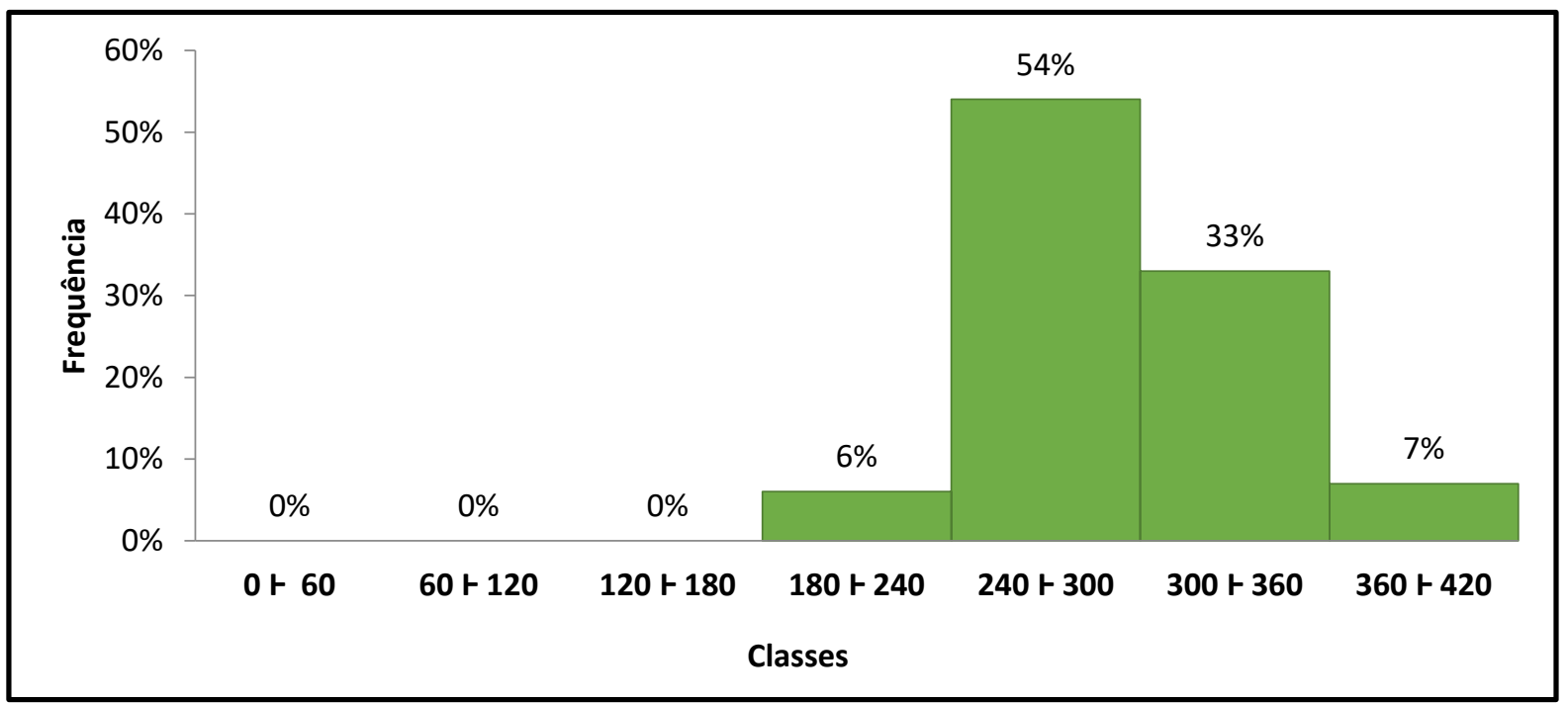

No abatedouro 3, observando-se os dados contidos na Tabela 11 e na Figura 12, nota-se que a maioria dos animais avaliados permaneceram na calha da sangria entre o intervalo de 2 a 3 min, sendo que cerca de $70 \%$ dos bovídeos abatidos saíram da calha durante a realização do processo de sangria, ou imediatamente após a mesma.

Figura 12 - Distribuição de frequência do tempo de permanência dos animais na calha da sangria, abatedouro 3.

\begin{tabular}{|c|c|c|}
\hline $\begin{array}{l}\text { Classes do Tempo de } \\
\text { Permanência na Calha }\end{array}$ & Frequência Absoluta & Frequência Relativa \\
\hline 0 F 60 & 0 & $0 \%$ \\
\hline 60 F 120 & 3 & $3 \%$ \\
\hline $120+180$ & 67 & $67 \%$ \\
\hline 180 F 240 & 21 & $21 \%$ \\
\hline $240+300$ & 9 & $9 \%$ \\
\hline TOTAL & 100 & $100 \%$ \\
\hline
\end{tabular}

Tabela 11 - Intervalos de permanência na calha da sangria, abatedouro 3.

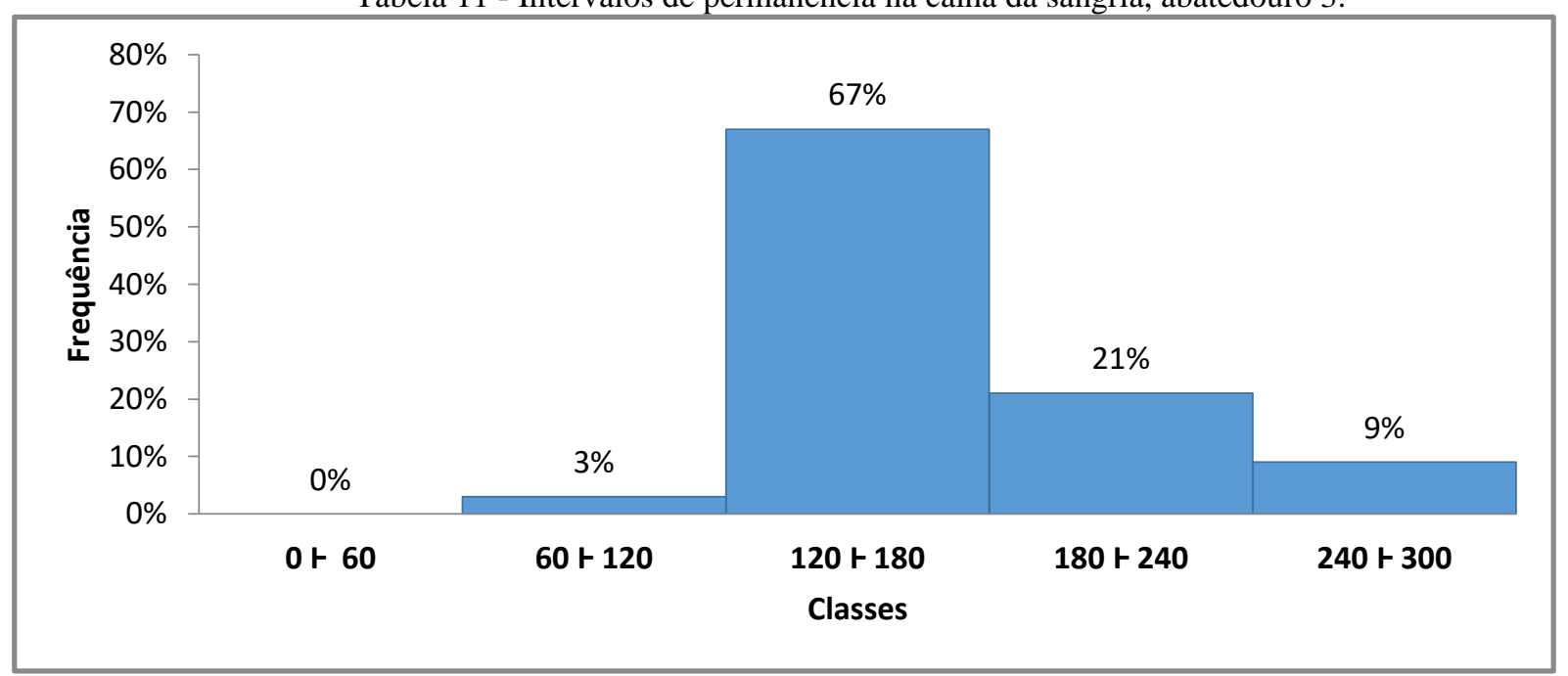


Segundo Roça et al. (2001), é preciso que cerca de $60 \%$ do volume total de sangue seja removido na sangria para poder se obter uma carne com adequada capacidade de conservação, sendo que o restante desse sangue fica retido nos músculos (10\%) e vísceras (20 a 25\%). O sangue tem pH alto $(7,35$ a 7,45), grande teor proteico e uma rápida putrefação, e com isso a capacidade de conservação de uma carne mal sangrada é limitada e constitui um problema de mau aspecto para o consumidor. Deste modo, a operação de sangria é considerada uma etapa muito importante no abate para se obter um produto de qualidade. Um tempo menor de sangria retém mais sangue nos músculos e consequentemente interfere na conservação e pode alterar o pH final da carne, por estar sujeita à proliferação bacteriana (LEITE et al., 2015).

\section{CONCLUSÕES}

Não há um programa específico de abate humanitário nos estabelecimentos visitados, onde observou-se que as falhas de manejo identificadas estão diretamente relacionadas à falta de treinamento dos colaboradores. Destaca-se a necessidade de capacitação dos mesmos sobre as legislações vigentes e sobre o tema bem-estar animal, bem como torna-se indispensável alterações estruturais que viabilizem a correta realização dos procedimentos e a redução do sofrimento desnecessário dos animais, o que interfere consequentemente na qualidade da carne fornecida na região. 


\section{REFERÊNCIAS}

ALVES, A. R.; FIGUEIREDO, J. P.; SANTANA, M. H.; ANDRADE, M. V.; LIMA, J. B. (2016). Efeito do Estresse Sobre a Qualidade de Produtos de Origem Animal. PUBVET, 10 (6), 448-459.

BERTOLONI, W.; ANDREOLLA, D. (2010). Eficácia do sistema de contenção (automatizado e mecânico) no atordoamento de bovinos. Ciência Rural, 40 (8), 1821-1827.

BRAGA, J. S.; MACITELLI, F.; LIMA, V. A.; DIESEL, T. (2018). O Modelo dos "Cinco Domínios" do Bem-estar Animal Aplicado em Sistemas Intensivos de Produção de Bovinos, Suínos e Aves. Revista Brasileira de Zoociências, 19 (2), 204-226.

BRASIL. MAPA. Inspeção de Carne Bovina. Padronização de Técnicas, Instalações e Equipamentos, 2007.

BRASIL. MAPA. Instrução Normativa $\mathrm{n}^{\circ} .3$, de 17 de janeiro de 2000. Regulamento técnico de métodos de insensibilização para o abate humanitário de animais de açougue. Diário Oficial da União, Brasília, DF, 24 jan. 2000. Seção 1, p.14-16.

CARLESCI, R. H.; BÜRGUER, K. P.; ROSSI, G. A.; SABA, R. Z.; MARTINS, A. M.; GONÇALEZ, P. O. (2014). Eficácia da Insensibilização em Bovinos pelo Uso de Pistola Pneumática de Penetração em Matadouro-frigorífico no Estado de São Paulo, Brasil. Revista Brasileira de Higiene e Sanidade Animal, $8(1), 73-80$.

DUARTE, F. H.; AFONSO, M. L. (2020). Abate de Animais Para o Consumo: Tese e Antítese do BemEstar Animal e dos Princípios da Medicina Veterinária. Brazilian Journal of Development, 6 (6), $35854-$ 35874.

GAllO, C.; GRANDIN, T.; TEUBER, C.; CARTES, M.; URIBE, H. (2003). Mejoras en la insensibilización de bovinos con pistola neumática de proyectil retenido tras cambios de equipamiento y capacitación del personal. Archivos de Medicina Veterinaria, 35 (2), 159-170.

GRANDIN, T. (2003). Good Management Practices for Animal Handling and Stunning. Dept. of Animal Science, Colorado State University. American Meat Institute Foundation. Disponível em: <https://www.aamp.com/documents/GMPsforAnimalHandling.pdf $>$. Acesso em: 15 de outubro de 2020.

GUERRERO, A.; VALERO, M. V.; CAMPO, M. M.; SAÑUDO, C. (2013). Some factors that affect ruminant meat quality: from the farm to the fork. Review. Acta Scientiarum. Animal Sciences, 35, 335347.

LANDIM, K. P. Eficiência do procedimento de insensibilização de bovinos por pistola de impacto sem penetração e o reflexo da qualidade da carne. 2011. 21f. Dissertação (Mestrado em Produção Animal) Universidade Camilo Castelo Branco.

LEITE, C. R.; NASCIMENTO, M. R.; SANTANA, D. O.; GUIMARÃES, E. C.; MORAIS, H. R. (2015). Influência do Manejo Pré-abate de Bovinos na Indústria Sobre os Parâmetros de Bem-estar Animal e Impactos no pH 24 Horas Post Mortem. Bioscience Journal, 31 (1), 194-203.

MÁRTIRE, R. V. Avaliação da Insensibilização em Bovinos de Abate em Três Matadouros Frigoríficos com Serviço de Inspeção Estadual no Paraná. 2016. 34f. Dissertação (Mestrado em Saúde e Produção de ruminantes) - Universidade do Norte do Paraná. 
NEVES, J. E. G. Influências de Métodos de Abate no Bem-estar e na Qualidade da Carne de Bovinos. 2008. 70f. Dissertação (Mestrado em Zootecnia) - Universidade Estadual Paulista "Júlio de Mesquita Filho", Faculdade de Ciências Agrárias e Veterinárias - Campus de Jaboticabal, São Paulo.

PERROTA, A. P. (2016). Abate Bovino e Rede Industrial: Um Estudo sobre a Introdução e Gestão Racional e Econômica das Emoções dos Animais. Política e Sociedade, 15 (33), 68-95.

PINTO, D. N. Avaliação da Tecnopatia na Insensibilização em Bovinos sobre os Parâmetros de Bem-estar Animal e Impactos na Cor e pH em Carcaças. 2018. 54f. Dissertação (Mestrado em Saúde e Produção de Ruminantes) - Universidade do Norte do Paraná.

ROÇA, R. O.; PADOVANI, C. R.; FILIPI, M. C.; SCHWACH, E.; UEMI, A.; SHINKAI, R. T.; BIOND, G. F. (2001). Efeitos dos métodos de abate de bovinos na eficiência da sangria. Ciência e Tecnologia de Alimentos, 21 (2), 244-248.

SOBRAL, N. C.; ANDRADE, E. N.; ANTONUCCI, A. M. (2015). Métodos de Insensibilização em Bovinos de Corte. Revista Científica de Medicina veterinária, 25 (25), 1-10. 